\title{
Coordination of subjects using a real practical case to boost learning results
}

\author{
Cristina Campos Sancho ${ }^{1}$, Angeles López ${ }^{2}$, Lledó Museros², Ismael Sanz ${ }^{2}$ \\ ${ }^{1}$ Departament de Llenguatges i Sistemes Informàtics, Universitat Jaume I, Spain, \\ ${ }^{2}$ Departament de Enginyeria i Ciència dels Computadors, Universitat Jaume I, Spain.
}

\begin{abstract}
In the European Higher Education Area (EHEA) the coordination of subjects presents a challenge and a key factor for students' learning and competence development. The joint planning of subjects about fundamentals of software engineering and design and implementation of information systems in computer science higher studies provides the students a comprehensive view of the development of a real information system from the conception of the idea to the final implementation for actual users. Such coordination aims to boost learning of the specific competences as well as the generic ones, and allows to generate synergies for students to obtain a direct benefit. This paper summarizes how this experience has been designed and carried out. We describe how to implement the coordination and in addition, we include the results of a survey conducted on students enrolled in the subjects the last academic year.
\end{abstract}

Keywords: learning improvement; teaching coordination; software engineering; practical case. 


\section{Introduction}

The current Bachelor's Degree in Computer Engineering at Universitat Jaume I, within the European Higher Education Area (EHEA), was launched in 2011. The total number of ECTS credits is 240 This degree offers four of the five sub-disciplines of computing science curricula (ACM/IEEE-CS, 2013) that can be chosen by students in the second half of the third year. All the students must acquire abilities related to the development of information systems, independently of the sub-discipline chosen. The subjects Software Engineering Fundamentals (SEF) and Design and Implementation of Information Systems (DIIS) are paramount in the learning and development of these abilities. The specific competences of both subjects 1 jointly provide the necessary skills for a graduate to develop a real information system from the conception of the idea to the final implementation for actual users. Temporally, their teaching is organized in the first and second half of the third academic year, respectively, and they have some competences in common and other ones that are complementary. The common competences are: i) Analysis and synthesis skills; ii) Ability to design, develop, select and evaluate computer applications and systems and to guarantee their reliability, security and quality in accordance with ethical principles and the laws and regulations in force; and iii) Ability to design and evaluate person-computer interfaces that ensure accessibility to and usability of computer systems, services and applications. The complete set of competences is detailed in the syllabus of the courses ${ }^{1}$.

To boost learning of these specific competences, and the generic competences as well, the faculty of these subjects collaborated to perform a joint learning, by defining a common practical case. This coordination presents a challenge and a key factor for students' learning and competences acquisition. Moreover, it provides a comprehensive view of the development of a real information system, because temporalization of subjects has a parallelism with actual projects in business. This paper summarizes how this experience has been carried out for the last five years and, in addition, we analyze information gathered from students that participated the last academic year to assess the result of this experience.

\section{Design of the coordination}

In this section we describe separately each subject emphasizing which are their specific learning outcomes, and what their challenges and risks are. Finally, we describe how we have designed and implemented the coordination experience as a teaching method using a practical case.

${ }^{1}$ http://ujiapps.uji.es/sia/rest/publicacion/2018/estudio/225 


\subsection{Standpoint of Software Engineering Fundamentals}

Software Engineering Fundamentals is a compulsory subject that, summarizing, includes as learning outcomes: acquiring knowledge about concepts as software engineering, computer systems, information systems, methodologies, techniques and software engineering support tools; identifying and analysing user requirements to define how a computer system can support organisations to achieve their goals; analysing and designing system processes and user interfaces; applying concepts as estimating, planning and managing software and finally writing technical documents on software engineering.

Teaching software engineering is amazing when students understand that the subject sets up the basis on which they will build the software product. But when we present the subject and the listed learning outcomes the students used to ask: where are we going to code?

In general, computer science students feel more comfortable sitting down in front of the computer for coding, debugging and testing, than talking to understand the businesses and processes, dealing with users, or developing accurate documentation and verifying and validating their proposals. They feel that this is not their job and that the methodological proposals have nothing to do with their actual job perspectives.

Therefore, to develop a strategy to make the subject of software engineering enjoyable was necessary. Furthermore, providing the coordination between a subject that mainly includes methodological concepts, with another one that puts into practice these concepts to develop a software product was a strategy that should provide better learning results.

\subsection{Standpoint of Design and Implementation of Information Systems}

Design and Implementation of Information Systems broadens knowledge acquired previously in the degree, related to the design of relational databases, software engineering fundamentals and the implementation of distributed systems. In this subject, students acquire knowledge about how to design, implement and evaluate user interfaces as well as the knowledge to develop web applications for access to information systems. This represents core abilities of a computer engineer, since the design and implementation of information systems has a very important role in enterprise software development.

The teaching of this subject is based mainly on two methodologies: project-based learning (PBL) (Dos Santos et al., 2009; Kay et al., 2000) and cooperative learning (CL). Through PBL the basic skills of the subject are taught by motivating students through solving a real problem, close to a real-world case that student may find in a workplace. Specifically, students undertake a project that involves the design and implementation of a web application.

Therefore, during one semester the students learn how to design a universal, usable, portable and easily to maintain web application, and at the same time all the technology needed to 
implement it. The analysis and design of the information system to implement is very important knowledge and task associated to DIIS, and trying to develop this task in the same semester seemed too ambitious. Thus, the coordination with Software Engineering Fundamentals seemed very beneficial for the subject, since the students will develop these analysis and design tasks during the first semester, before this subject starts.

\subsection{Coordination planning and implementation}

The curricula of the EHEA Bachelor's Degree in Computer Engineering promote to coordinate subjects as a key issue to improve student learning results. Regarding the common contents, the problems detected and organizational aspects, these two subjects were considered to be good candidates for such a coordination. Next we detail the steps followed to implement the coordination.

\section{Set up the coordination goals}

In SEF it was important to motivate the students to develop the first phases of the project. Therefore to describe the project as a preliminary study of a software product could produce a positive effect and a change of attitude. In DIIS the main goal of the coordination was to start with a detailed analysis of the case already performed. Also, working in teams is important in both.

\section{Define actions to implement the coordination.}

There are several mechanisms to implement coordination for improving learning as for instance Clemmensen and Nørbjerg (2004). After studying different options we decided to use a real practical case as the guiding theme in the coordination of both subjects to provide continuity and completeness of their learning objectives. The teaching coordination was performed through the following actions:

- Define a practical case based on a real information system.

- Promote and highlight as an added value the use of a unique practical case in both subjects.

- Meetings to coordinate and track the project development.

Before the academic year starts, the faculty prepares the case description accurately in order to be understandable for students, considering that the resolution of the problem requires acquiring the abilities of the subjects but also with an equilibrated level of difficulty. The case is different and renewed every year to show novel aspects that make it more motivating for the students (Anderson and Schiano, 2014). Figure 1 shows the contents that the students develop to solve the proposal of the practical case in each subject and those that are common in both. 
To employ effective strategies to enhance student motivation will provide better engagement and good effects on student performance (Liu et al, 2012; Domenech et al, 2015). During the first semester, the students apply the concepts acquired in SEF to carry out the first phases of a software engineering project which aims to develop an information system. The result is a report where they describe the objectives and goals of the product, the requirements and the analysis and a high level design of the information system. During the second semester, the students design and implement the system, beginning from the previous work. In this case, the results are a deeper design, including usability issues, and the coding of the final information system. This use of a common case provides benefits to both, students and faculty, because the effort in the comprehension of the case can be profitable during all the academic year. We need to conveniently explain this fact to increase students' awareness of the importance of doing a good job in SEF to obtain good results in DSII. If a student is not enrolled in both subjects the same academic year, she is included in a group where the basic knowledge needed about the case is guaranteed by the other team members.

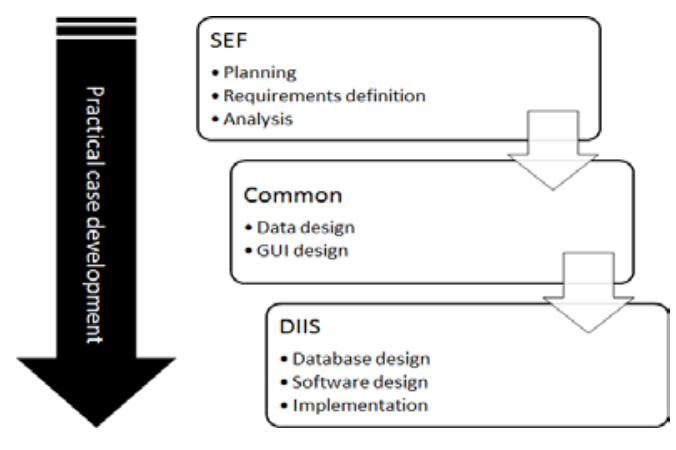

Figure 1. Subject contents of the practical case development.

The faculty develop at least three meetings to organize the coordination: the first one, at the beginning of the first semester, to review the practical case definition and setup the minimum requirements of the solution; the second one, at the end of the first semester to track the work that the students have performed, to share results, to identify changes and to establish the common basis of the design that is considered as an starting point for the second semester; a final meeting is conducted to assess the complete results of the coordination and the practical case, at the end of the academic year. These meetings aim to share results of the implementation of the project in the subjects, to identify problems and to suggest solutions.

\section{Coordination assessment.}

Each year we review the work done by the students and the problems identified. This analysis provides a feedback that is used to improve how the faculty coordinate their subjects' contents as well as to define the new practical case for the next academic year. During several academic years, we have carried out this assessment regarding the academic results and how 
the students have been involved in the subject. The last year we conducted a survey to gather information from students and to assess what are the strengths and weaknesses of this coordination work. The work developed to conduct this survey is described and analyzed in the next section.

\section{Survey}

In order to validate whether the coordination of these two subjects provide benefits and better results we planned to develop a survey to collect information from students that had already finalized both subjects. Therefore we used a questionnaire as a primary mean and we completed the information gathering by interviewing a small group of students (Fink, 2003).

The steps we followed to develop this survey are:

- Survey goal definition and plan: to know what is the actual students' perception about the coordination, to assess whether the coordination provide acceptable results and to detect any need of improvement.

- Information gathering design: anonymous questionnaires and interviews. We defined direct questions that were easy to answer to motivate students to participate.

- Questionnaire application and interviews

- Data collection and analysis.

Next, the results of the questionnaire and the interviews are described and analyzed.

\subsection{Questionnaire and Results}

For gathering the students' opinion we prepared a questionnaire which was delivered to all the students enrolled in the subjects during academic year 2017-2018. The number of potential addressees are 60, answers 19; answers from students enrolled in one subject 3, and answers from students enrolled in both subjects 16 .

The first question asked for the enrollment in both subjects the same academic year, with answer yes/no. Then, a number of statements were provided, together with a Likert-type scale with 5 possible responses from "strongly disagree" to "strongly agree". The students who had enrolled the subjects in different academic years where asked about the difficulty of understanding the practical case at DIIS. They all agreed that it was difficult to understand the practical case at DIIS even that the teachers provided a standard solution. One of them even strongly agreed. The other students, who had enrolled in both subjects the same academic year, were asked about their agreement or disagreement to these four statements:

- S1: The solution developed in SEF provided me a better idea of the problem to be solved in the implementation 
- S2: While attending DIIS, I modified the model performed in SEF because as we progress in the case implementation I have a better understanding of the problem.

- S3: The fact of coordinating the practical case in both subjects is a positive aspect for integration of the knowledge that we acquire.

- S4: The transition from one subject to the other has been adequate.

Figure 2 shows the assessments for these four sentences. Most students agree that the solution developed in SEF provided them a better understanding of the problem during the implementation (S1). Also, most students agree that they modified the model performed in SEF due to a better understanding of the problem, but such agreement was not strong (S2). Maybe this is due to the fact that these modifications usually consist of minor changes. Nevertheless, almost three quarters of respondents agree that the coordination is positive for the integration of knowledge (S3), and most of them agree strongly. Finally, most of the students consider that the transition was adequate (S4).

S1

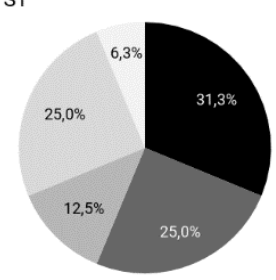

Strongly agree
S2

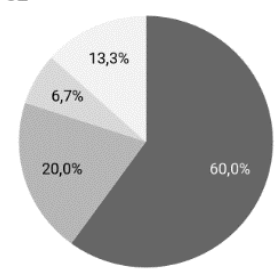

S3

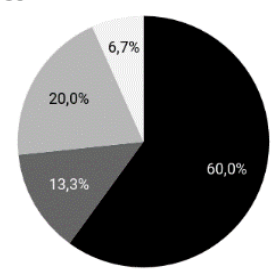

Disagree
S4

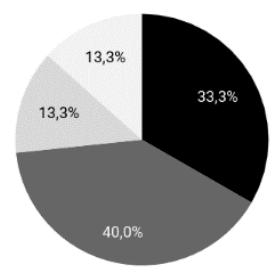

Strongly disagree

Figure 2. Degree of agreement of students that performed both subjects in one academic year, about coordination of both subjects and transition between them. The total count does not include non-respondents. Most students agree that the transition is adequate (S4), and coordination is positive (S3). Although not so strongly, most students agree that coordination helps to know better the practical case to be developed (S1 and S2).

Finally, the questionnaire included an invitation to comment issues they missed:

- M1: Did you miss any issue or topic in SEF? (Yes/no). If yes, which?

- M2: Did you miss any issue or topic in DIIS? (Yes/no). If yes, which?

Most answers were no: $100 \%$ answered no to M1, while $75 \%$ answered no to M2. All of the $25 \%$ positive answers asked for extensions of the current examples and technologies used in DIIS, but none of the answers referred to neither the coordination, nor the planning and approach of the practical case. 


\subsection{Summary of interviews}

We planned to interview a representative small group of students in order to elicit information about aspects that can be improved in the teaching coordination. To summarize, students consider the coordination experience using a practical case as a positive aspect in the learning activities of both subjects, mainly because the effort in understanding a real case is worth when the case is used by two subjects. From these interviews we concluded some key issues that must be considered to be improved, which are the difficulty of the practical case and the organization of the student teams.

\section{Discussion and conclusions}

After these academic years, the faculty involved in these subjects have acquired a collaborative synergy that improves not only the teaching planning and performance, but also the student's perception of the learning contents completeness. Both subjects are complementary and this coordination helps students to acquire a complete view of a software engineering project. The survey has provided a good feedback to be taken into account to improve the teaching design, mainly when it comes to define the practical case. For next years, we will review the statements and we will foster students to participate.

The results encourage us to continue and to improve this coordination because it is a primordial tool to successfully reach the learning outcomes defined in both subjects. As future work we plan to publish a solved real case for showing the complete process of developing a web based information system, starting by the requirements collection of the case and finishing with the user interface evaluation of the resulting system.

\section{References}

ACM/IEEE-CS (2013) Computer Science Curricula 2013: Curriculum Guidelines for Undergraduate Degree Programs in Computer Science. The Joint Task Force on Computing Curricula Association for Computing Machinery (ACM) and IEEE Computer Society. https://www.acm.org/binaries/content/assets/education/cs2013_web_final.pdf

Anderson, E.; Schiano, B. (2014) Teaching with Cases: A Practical Guide. Harvard Business Review Press.

Clemmensen, T. C. ; Nørbjerg J. (2003) Separation in theory, coordination in practice teaching HCI and SE. Special Issue: Bridging the Process and Practice Gaps Between Software Engineering and Human Computer Interaction. pp.- 99-110.

Domenech, J., Blazquez, D., de la Poza, E., \& Muñoz-Miquel, A. (2015). Exploring the impact of cumulative testing on academic performance of undergraduate students in Spain. Educational Assessment, Evaluation and Accountability, 27(2), 153-169. 
Dos Santos, S. C., Moraes Batista, M. da Conceição, Cavalcanti, A. P. C., Albuquerque, J. O. and Meira, S. R. L., Applying PBL in Software Engineering Education, 22nd Conference on Software Engineering Education and Training, 2009, pp. 182-189.

Fink, A. (2003). The survey handbook. Thousand Oaks, CA: SAGE Publications, Inc.

Kay, J., Barg, M., Fekete, A., Greening, T., Hollands, O., Kingston, J.H. and Crawford, K., (2000) Problem-Based Learning for Foundation Computer Science Courses, Computer Science Education, 10:2, 109-128,

Liu, O. L., Bridgeman, B., \& Adler, R. M. (2012). Measuring Learning Outcomes in Higher Education: Motivation Matters. Educational Researcher, 41(9), 352-362.

Robbins, N.B.; Heiberger, R.M. (2011). Plotting Likert and Other Rating Scales. JSM Proceedings, Section on Survey Research Methods. American Statistical Association. pp. 1058-1066. 